\title{
Generalizing Refinement Operators to Learn Prenex Conjunctive Normal Forms
}

\author{
Shan-Hwei Nienhuys-Cheng, Wim Van Laer \\ Jan Ramon, Luc De Raedt \\ cheng@few.eur.nl,\{wimv,janr,lucdr\}@cs.kuleuven.ac.be \\ Department of Computer Science, Katholieke Universiteit Leuven \\ No Institute Given
}

\begin{abstract}
Inductive Logic Programming considers almost exclusively universally quantified theories. To add expressiveness we should consider general prenex conjunctive normal forms which use existential variables. Learning with refinement operators is the most used learning method in ILP. To extend refinement operators to PCNF, we should first extend substitutions to PCNF. The usual substitutions often generalize a formula instead of specializing it because of the existential variables. In this article we define substitutions to specialize a given PCNF and a weakly complete downward refinement operator. Based on this operator, we have implemented a simple learning system PCL on some type of PCNF.
\end{abstract}

\section{Introduction}

Inductive Logic Programming learns a correct logic formula with respect to examples. The definition of correctness has to do with the way examples are presented. Suppose some interpretations are given as examples, formulas with these examples as models should be found. A downward refinement operator $\rho$ can be used to search such a formula. If a searching process begins with $T=\{\square\}$, it should be replaced by the set of its refinements in $\rho(T)$ because $\square$ has no models. A refinement $\phi \in \rho(T)$ may have to be replaced by its refinements again because some given interpretations are not its models. This process can go on until correct formulas are found.

Refinement operators have often been used ([S81,RD97]) to learn a correct universally quantified theory incrementally. If clause $C$ subsumes clause $D$, then a refinement chain exists from $C$ to $D$ using elementary substitutions and adding literals. Let $C=p(x, y)$ and $D=p(x, x) \vee \neg q(f(x))$. Then a chain may be $p(x, y), p(x, y) \vee \neg q(z), p(x, y) \vee \neg q(f(u)), p(x, x) \vee \neg q(f(u)), p(x, x) \vee \neg q(f(x))$. If a correct universally quantified theory does exist, then a refinement chain from $\square$ to every clause in this theory exists because $\square$ subsumes every clause.

Until now almost all ILP researchers are only interested in universally quantified clauses, especially definite program clauses. However, we may want to learn a concept expressed by a formula $\phi$ with existential variables such as a prenex conjunctive normal form (PCNF). To solve such problems, one considers the universally quantified Skolem standard form $\psi$ of $\phi$. It is well known 
that $\psi \models \phi$ but not $\phi \models \psi$. For instance, let the 3 -ary predicate $p$ be interpreted in the set of real numbers $\mathbf{R}$ as $p(x, y, z)$ is true iff $x y=z$. The concept that for an arbitrary $z \in \mathbf{R}$, there are $x, y$ such that $x y=z$ can be expressed by $\phi=\forall z^{2} \exists x \exists y p(x, y, z)$. A standard form of the formula $\phi$ is $\psi=\forall z p(f(z), g(z), z)$ for new function symbols $f, g$. A model of $\phi$ is also a model of $\psi$ only when $f$ and $g$ are interpreted in certain ways, e.g. $f(z)=2 z$ and $g(z)=z / 2$, but we would like to check the truth value of $\phi$ directly. In a database we may have an integrity constraint $\forall x \forall y \exists z \neg \operatorname{sell}(x, y) \vee \operatorname{suppl} y(x, y, z)$ : if a shop $x$ sells an item $y$, there must be a company $z$ which supplies $x$ with $y$. Of course we can define one particular supplier as $f(x, y)$ but we have to change $f$ when we consider another supplier. To add expressiveness we should consider learning PCNF in general.

We would also like to use (elementary) substitutions to refine a PCNF. The usual substitutions often generalize a formula instead of specializing it because of the existential variables. Let $\phi=\forall x \exists y p(x, y)$ and $\psi=\forall x p(x, x)$. Then $\psi \models \phi$ but $\phi \not \forall \psi$. Therefore we will define a new type of substitutions to specialize a PCNF. Based on our substitutions and adding literals, we can define a downward refinement operator $\rho$ which is weakly complete: For every $\phi$ there is a refinement chain from $T$ to $\phi$, i.e., there is an $n$ such that $\phi \in \rho^{n}(\top)$.

Generalizing and specializing a formula with existential quantifiers have also been considered in [GF96]. A formula there involves only one clause (actually a clause presented in a special form). The variables in the head of a clause are universally quantified. The variables not in the head are quantified separately in the body by existential and universal quantifiers. Some rules are given to manipulate the variables only in the body. It seems that the rules are motivated by the following principle: if the body is generalized, then the formula is specialized and vice versa. [GF96] adopts neither PCNF in general nor a uniform approach with substitutions.

In this article we begin with establishing some properties of PCNF. We then define (elementary) substitutions and a downward refinement operator. We use a refinement operator to search in the set of theories expressed in PCNF. This differs from a classic refinement opertor which is defined in a serach space of universally quantified clauses. Most proofs will be omited (see [NLR99]). We explain then briefly our first step in implementation. If $I_{1}, I_{2}, \ldots, I_{n}$ is a set of interpretations, the system PCL finds a PCNF $\phi$ such that every $I_{j}$ is a model of $\phi$. Hence we have generalized the Claudien system[RD97] which only deals with the standard forms.

\section{Prenex Conjunctive Normal Forms}

In the first subsection we give some well known basic definitions and results in logic (see [NW97]). In the second subsection we state two important lemmas of ours. In the last subsection we consider the effects of adding literals to a clause in a PCNF. 


\subsection{Preliminaries}

Definition 1. An interpretation $I$ with domain $D$ of a first-order language $L$ consists of the following: (a) Each n-ary function symbol $f$ in $L$ is assigned a mapping from $D^{n}$ to $D$. (b) Each $n$-ary predicate symbol $p$ in $L$ is assigned a mapping from $D^{n}$ to $\{$ true, false $\}$.

Definition 2. Let I be an interpretation of the first-order language $L$ with domain D. Let $V$ be a set of variables, then a mapping $\theta: V \rightarrow D$ is called a variable assignment from $V$. Given a variable assignment $\theta$ from the set of variables in a formula $\phi$, we can check if $\phi$ is true or false under $I$ and $\theta$. If $\phi$ is a closed formula, then the truth value of $\phi$ is independent of the variable assignment we choose. I is a model of the closed formula $\phi$ if $\phi$ is true in I. Formula $\psi$ logically implies formula $\phi$, denoted by $\psi \models \phi$, if every model of $\psi$ is also a model of $\phi . \psi$ and $\phi$ are called logically equivalent, denoted by $\psi \Leftrightarrow \phi$, if they have the same models.

Definition 3. A clause is a disjunction of a finite number of literals. A prenex conjunctive normal form (PCNF) $\psi$ is a closed formula $q_{1} x_{1} q_{2} x_{2} \ldots q_{n} x_{n}\left(C_{1} \wedge\right.$ $\left.C_{2} \wedge \ldots \wedge C_{m}\right)$ where $q_{i}$ is a quantifier $(\exists$ or $\forall), x_{i} \neq x_{j}$ if $i \neq j$ and $C_{j}$ is a clause. $q_{1} x_{1} q_{2} x_{2} \ldots q_{n} x_{n}$ is the prenex of $\psi$ and $C_{1} \wedge C_{2} \wedge \ldots \wedge C_{m}$ is the matrix of $\psi$. We denote $\psi$ often by $q_{1} x_{1} q_{2} x_{2} \ldots q_{n} x_{n} M\left(x_{1}, \ldots, x_{n}\right)$ or $Q\left(x_{1}, \ldots, x_{n}\right) M\left(x_{1}, \ldots, x_{n}\right)$ or $Q(\bar{x}) M(\bar{x})$ or $Q(\psi) M(\psi)$ or $Q M$. We call variables in $Q(\psi)$ universal or existential; depending on how they are bound; the set of existential and universal variables are denoted by eVar $(\psi)$ and $u \operatorname{Var}(\psi)$, respectively. We have $\operatorname{Var}(\psi)=u \operatorname{Var}(\psi) \cup \mathrm{e} \operatorname{Var}(\psi)$.

Note that if $\left\{y_{1}, \ldots, y_{m}\right\} \supseteq\left\{x_{1}, \ldots, x_{n}\right\}$, then $Q(\bar{y}) M(\bar{x}) \Leftrightarrow Q(\bar{x}) M(\bar{x})$.

Theorem 1. Let $\phi$ be a closed formula. Then there exists a PCNF $\psi$ such that $\phi$ and $\psi$ are logically equivalent.

\subsection{Some properties of PCNF}

We often need to check if some interpretation $I$ is a model of a formula $\psi$. Lemma 1 in this subsection gives a necessary and sufficient condition for $I$ to be a model of $\psi$. Lemma 2 tells that the truth value of a formula has often to do with the positions of variables in the prenex. Both lemmas will often be used for proving other results.

Example 1. Let $\psi=\exists x \forall y p(x, f(y))$ and $\phi=\forall y \exists x p(x, f(y))$. Then $\psi \models \phi$ : Let $I$ be an interpretation with domain $D$. Then $\psi$ is true in $I$ iff there is a $d \in D$ such that the formula $\forall y p(d, f(y)))$ is true in $I$ iff there is $d \in D$ such that for every $e \in D, p(d, f(e))$ is true. On the other hand, $\phi$ is true in $I$ iff for every $e \in D, \exists x p(x, f(e))$ is true iff for every $e \in D$, there is $d \in D$ such that $p(d, f(e))$ is true. Notice that the choice of $d$ here may depend on $e$. Since $I$ is a model of $\psi$, for every $e \in D$, we can choose the same $d \in D$ such that $p(d, f(e))$ is true in $I$. Consider the Herbrand interpretation $I=\{p(t, f(t)) \mid t$ ground $\}$. Then $I$ is 
a model of $\phi$. For an assignment $\sigma=\{x / t\}$, let $\gamma=\{y / t\}$ then $\phi(\sigma \cup \gamma)$ is true. We can not say $I$ is a model of $\psi$ because $\gamma$ depends on $\sigma$. We can generalize this example to the following lemmas

Lemma 1. Let $\phi=q_{1} x_{1} \ldots q_{n} x_{n} M\left(x_{1}, \ldots, x_{n}\right)$. An interpretation $I$ with domain $D$ is a model of $\phi$ iff for every $\sigma: u \operatorname{Var}(\phi) \rightarrow D$ there is a $\gamma: \operatorname{eVar}(\phi) \rightarrow D$ such that the following two conditions are satisfied: $(a) M(\sigma \cup \gamma)$ is true in I. (b) the definition of $\gamma$ on $x_{i}$ depends only on how $\sigma$ and $\gamma$ are defined on $\left\{x_{1}, x_{2}, \ldots, x_{i-1}\right\}$, i.e., an element in $D$ can be assigned to $x_{i}$ after the assignment of all $x_{j}, j<i$ has been done.

Lemma 2. Let

$$
\psi=q_{1} x_{1} \ldots \exists x_{i} \ldots \forall x_{j} \ldots q_{n} x_{n} M, \phi=q_{1} x_{1} \ldots \forall x_{j} \ldots \exists x_{i} \ldots q_{n} x_{n} M
$$

and suppose there is no other existential variable between $x_{i}$ and $x_{j}$ in the prenexes of these two formulas. Then $\psi \models \phi$.

Proof . Suppose $I$ is a model of $\psi$. We want to prove that $I$ is also a model of $\phi$ by applying Lemma 1. Let $\sigma: u \operatorname{Var}(\phi) \rightarrow D$. By Lemma 1, there is a variable assignment $\gamma$ of $\operatorname{eVar}(\psi)$ such that $M(\psi)(\sigma \cup \gamma)$ is true under $I$. The assignment of $x_{k} \in \operatorname{eVar}(\psi)$ may depend on the definition of $\sigma$ and $\gamma$ on variables before $x_{k}$ in $Q(\psi)$. Since $u \operatorname{Var}(\psi)=u \operatorname{Var}(\phi)$ and $M(\psi)=M(\phi)$, we have $M(\phi)(\sigma \cup \gamma)$ true under $I$. By Lemma 1, we can say $I$ is a model of $\phi$ if $\gamma$ in $x_{k} \in \operatorname{eVar}(\phi)$ depends on the assignment of variables before $x_{k}$ in $Q(\phi)$. Note that $Q(\phi)$ interchanges only the order of $\exists x_{i}$ and $\forall x_{j}$ in $Q(\psi)$. The assignment of $x_{i}$ in $\gamma$ depends only on the assignment of $\sigma, \gamma$ on $x_{1}, \ldots, x_{i-1}$ which appear before $\exists x_{i}$ in $Q(\phi)$. If $x_{k} \in \mathrm{e} \operatorname{Var}(\phi)$ is another existential variable, then it is not between $x_{i}$ and $x_{j}$ so $x_{1}, \ldots, x_{k-1}$ are still before $x_{k}$ in $Q(\phi)$. That means $I$ is a model of $\phi$.

\section{$2.3 \quad$ Adding literals}

A classic refinement step for a universal quantified PCNF extends a clause in the matrix with an extra literal containing new variables. This will also be done for our refinement operator for PCNF. For this we need the the following results which are based on the fact that the disjunctions of formulas is true if at least one of them is true.

Lemma 3. Let $\psi=q_{1} x_{1} \ldots q_{n} x_{n} C_{1} \wedge C_{2} \ldots \wedge C_{m}$ where every $C_{i}$ is a clause. Let $L$ be a literal which contains only variables $y_{1}, \ldots, y_{k}$ that are new w.r.t. $\psi$. If $\phi=q_{1} x_{1} \ldots q_{n} x_{n} \forall y_{1} \ldots \forall y_{k} C_{1} \wedge \ldots \wedge\left(C_{j} \vee L\right) \ldots \wedge C_{m}$, then $\psi \models \phi$.

We call the action in Lemma 3 adding a u-literal. Similarly we can prove the following lemma which adds an e-literal to a formula.

Lemma 4. Let $\psi=q_{1} x_{1} \ldots q_{n} x_{n} C_{1} \wedge C_{2} \ldots \wedge C_{m}$ where every $C_{i}$ is a clause. Let $L$ be a literal which contains only new variables $y_{1}, \ldots, y_{k}$ that are new w.r.t. $\psi$. If $\phi=\exists y_{1} \ldots \exists y_{k} q_{1} x_{1} \ldots q_{n} x_{n} C_{1} \wedge \ldots \wedge\left(C_{j} \vee L\right) \ldots \wedge C_{m}$, then $\psi \models \phi$. 


\section{Substitutions and Specializations}

We are used to it that a substitution $\theta$ is defined by replacing some variables with terms. For a universally quantified clause $C$ we have always $C \models C \theta$. This is not valid for PCNF as the following examples show. Thus we are motivated to define a new type of substitutions which specialize PCNF.

Example 2. Consider the following implications:

$$
\begin{aligned}
& \forall x p(x) \models p(a) \text { and } p(a) \forall \forall x p(x) \\
& p(a) \models \exists x p(x) \text { and } \exists x p(x) \not p p(a) \\
& \exists x p(x, x) \models \exists x \exists y p(x, y) \text { and } \exists x \exists y p(x, y) \forall \exists x p(x, x)
\end{aligned}
$$

A unification of two universally quantified variables does not always specialize a PCNF. Let $\psi=\forall x \exists y \forall z p(x, y, z), \phi=\forall x \exists y p(x, y, x), \phi^{\prime}=\exists y \forall z p(z, y, z)$, and $I=\left\{p\left(t, f(t), t^{\prime}\right) \mid t, t^{\prime}\right.$ ground $\}$. Then $I$ is a model of $\psi$ and $\phi$ but not a model of $\phi^{\prime}$. For $\phi^{\prime}$ true in $I$, we need an $s$ such that $p(t, s, t)$ true for every $t$.

\subsection{Elementary substitutions for PCNF}

A matrix can be pictured as a tree, with the root on top. At each node, number downgoing branches 1, 2, 3, etc. from left to right. Each node and the tree hanging from it is given by the path that leads to it from the top. For example, let $M=p(x, y) \wedge(p(x, x) \vee \neg q(f(x)))$. The second clause has position $\langle 2\rangle . \neg q(f(x))$ has position $\langle 2,2\rangle, f(x)$ has position $\langle 2,2,1\rangle$, etc.

Definition 4. An substitution for a matrix $M$ has the form $\theta=\left\{\left(t_{1} / s_{1}, p_{1}\right), \ldots\right.$, $\left.\left(t_{n} / s_{n}, p_{n}\right)\right\}$. Me is a matrix formed by using $M$ and $\theta$ : for every $i$, the term at position $p_{i}$ in $M$ is $t_{i}$ and $t_{i}$ should be replaced by $s_{i}$. For example, if $M=$ $p(x, y) \wedge(p(x, x) \vee \neg q(f(x)))$ and $\theta=\{(x / f(z),\langle 1,1\rangle),(f(x) / g(z),\langle 2,2,1\rangle)\}$, then $M \theta=p(f(z), y) \wedge(p(x, x) \vee \neg q(g(z)))$. It is easy to see that the old definition of a substitution is a special case of the new kind of substitutions. In such a case we use the old notation where the positions are not needed.

Definition 5. Let $\psi=q_{1} x_{1} \ldots q_{n} x_{n} C_{1} \wedge \ldots \wedge C_{m}=Q(\psi) M(\psi)$ be a PCNF. There are the following 5 types of elementary substitutions for $\psi$ :

The first two types have to do with universal variables:

- Let $x_{i}, x_{j} \in \mathrm{u} \operatorname{Var}(\psi)$ and $i<j$. An elementary u-unification $\theta=\left\{x_{j} / x_{i}\right\}$ for $\psi$ can be applied to $\psi$ such that $\psi \theta=q_{1} x_{1} \ldots q_{n} x_{n}(M(\psi) \theta)$. For example let $\psi=\forall x \forall y p(f(x), y)$ and $\theta=\{y / x\}$. Then $\psi \theta=\forall x \forall y p(f(x), x)$ which is equivalent to $\forall x p(f(x), x)$.

- Let $x_{i} \in \mathrm{u} \operatorname{Var}(\psi)$. If $t=f\left(y_{1}, \ldots, y_{k}\right)$, where $y_{1}, \ldots, y_{k}$ are new distinct variables w.r.t. $\psi$, then $\theta=\left\{x_{i} / t\right\}$ is called an elementary u-substitution. Let $\psi \theta$ be the new formula constructed as follows. All $x_{i}$-occurrences in the matrix of $\psi$ are replaced by $t$ simultaneously, i.e. $M(\psi \theta)=M(\psi) \theta$. Moreover, the $\forall x_{i}$ in the prenex of $\psi$ is replaced by $\forall y_{1} \forall y_{2} \ldots \forall y_{k}$. For example, let $\psi=\forall x \exists y p(x, y)$ and $\theta=\{x / f(u, v)\}$. Then $\psi \theta=\forall u \forall v \exists y p(f(u, v), y)$. 
The next two types have to do with existential variables:

- Let $x_{i} \in \mathrm{e} \operatorname{Var}(\psi)$ and let $\left\{\left(x_{i}, p_{1}\right), \ldots,\left(x_{i}, p_{k}\right)\right\}$ be a proper subset of the $x_{i}$ occurrences in $M(\psi)$. If $z$ is a new variable, then $\theta=\left\{\left(x_{i} / z, p_{1}\right), \ldots\right.$,

$\left.\left(x_{i} / z, p_{k}\right)\right\}$ is called an elementary e-antiunification and $\psi \theta$ is the PCNF $q_{1} x_{1} \ldots \exists x_{i} \exists z q_{i+1} x_{i+1} \ldots q_{n} x_{n}(M \theta)$. For example, let $\psi=\exists x p(x, x)$ and $\theta=$ $\{x / z,\langle 2\rangle\}$. Then $\psi \theta=\exists x \exists z p(x, z)$.

- Let $t=f\left(x_{i_{1}}, \ldots, x_{i_{m}}\right)$ which contains only distinct existential variables. Let $i_{j}=\max \left\{i_{1}, \ldots, i_{m}\right\}$. Let $\left\{\left(t, p_{1}\right), \ldots,\left(t, p_{k}\right)\right\}$ be occurrences in $M(\psi)$. If $z$ is a new variable, then $\theta=\left\{\left(t / z, p_{1}\right), \ldots,\left(t / z, p_{k}\right)\right\}$ is called an elementary esubstitution for $\psi$. We define $\psi \theta=q_{1} x_{1} \ldots q_{i_{j}} x_{i_{j}} \exists z q_{i_{j}+1} x_{i_{j}+1} \ldots q_{n} x_{n}(M \theta)$. For example, let $\psi=\forall x \exists y \exists u \exists v p(x, u) \wedge(p(x, y) \vee \neg q(f(u, v)))$. If $\theta=$ $\{(f(u, v) / z,\langle 2,2,1\rangle)\}$, then $\psi \theta=\forall x \exists y \exists u \exists v \exists z \quad p(x, u) \wedge(p(x, x) \vee \neg q(z))$.

The last type has to do with interchanging an existential with a universal variable:

- Suppose $x_{i} \in \mathrm{eVar}(\psi), x_{j} \in \mathrm{u} \operatorname{Var}(\psi)$ and $i<j$. If there is no other existential variable between $x_{i}$ and $x_{j}$ in $Q(\psi)$, then $\left\{\left(x_{i}, x_{j}\right)\right\}$ denotes an elementary eu-substitution. It interchanges the positions of $x_{i}$ and $x_{j}$ in $Q(\psi)$. For example, let $\theta=\{(x, y)\}$. Then $(\exists x \forall y p(x, y)) \theta=\forall y \exists x p(x, y)$.

\subsection{Substitutions and specializations}

For every elementary substitutions $\theta$ w.r.t. $\psi$ we can prove that $\psi \models \psi \theta$. For instance,

Lemma 5. Let $\psi=Q(\psi) M(\psi)$ be a PCNF. Let $\theta=\left\{\left(t / z, p_{1}\right), \ldots,\left(t / z, p_{m}\right)\right\}$ be an elementary e-substitution. Then $\psi \models \psi \theta$.

Proof . Let $I$ be a model of $\psi$. Let $t$ contain only the existential variables $x_{i_{1}}, \ldots, x_{i_{k}}$ where $i_{1}<i_{2} \ldots<i_{k}$. Then $\psi \theta=q_{1} x_{1} \ldots q_{i_{k}} x_{i_{k}} \exists z q_{i_{k}+1} x_{i_{k}+1}$ $\ldots q_{n} x_{n} M \theta . M(\psi)$ differs from $M(\psi \theta)$ only at position $p_{1}, p_{2}, \ldots, p_{k}$. Clearly, $\mathrm{u} \operatorname{Var}(\psi \theta)=\mathrm{u} \operatorname{Var}(\psi)$ and $\mathrm{e} \operatorname{Var}(\psi \theta)=\mathrm{e} \operatorname{Var}(\psi) \cup\{z\}$. We want to prove for every variable assignment $\sigma$ of $u \operatorname{Var}(\psi \theta)$ to $D$, there is a variable assignment $\gamma$ of $\operatorname{eVar}(\psi \theta)$ to $D$ such that $M(\psi) \theta(\sigma \cup \gamma)$ is true under $I$. Since $I$ is a model of $\psi$, and the assignment $\sigma$ is also an assignment of $u \operatorname{Var}(\psi)$, by Lemma 1 there is a variable assignment $\gamma$ of $\operatorname{eVar}(\psi)$ to $D$ such that $M(\psi)(\sigma \cup \gamma)$ is true under $I$. Moreover, if $x_{i} \in e \operatorname{Var}(\psi)$, then $\gamma\left(x_{i}\right)$ depends only on how $\sigma$ and $\gamma$ beheave on variables before $x_{i}$ in $P(\psi)$. Notice that at every position $p_{j}$ in $M(\psi)(\sigma \cup \gamma)$ we have in fact $t \gamma$. We consider the subsitution $\gamma^{\prime}=\gamma \cup\{z / t \gamma\}$. Then $M(\psi)(\sigma \cup \gamma)=M(\psi) \theta\left(\sigma \cup \gamma^{\prime}\right)$. Notice $z$ is behind all variables $x_{1}, \ldots, x_{i_{k}}$ in $P(\psi \theta)$. Thus $z$ depends also only on how $\sigma$ and $\gamma^{\prime}$ defined on the variables before it. By applying Lemma 1, we have $I$ is a model of $\psi \theta$.

We can combine the elementary substitutions to get the following definition and theorem. 
Definition 6. Let $\psi$ be a PCNF. Suppose $\theta_{1}$ is an elementary substitution w.r.t. $\psi$ and $\theta_{i}$ is elementary w.r.t. $\left(\ldots\left(\psi \theta_{1}\right) \theta_{2} \ldots\right) \theta_{i-1}$ for every $i=2, \ldots, n$. Let $\theta=\theta_{1} \ldots \theta_{n}$ be the composition of these $\theta_{i}$, i.e. $\left.\psi \theta=\left(\ldots\left(\psi \theta_{1}\right) \theta_{2}\right) \ldots\right) \theta_{n}$. Then $\theta$ is called a substitution w.r.t. $\psi$.

Theorem 2. For a PCNF $\psi$ and a substitution $\theta$ defined as above, $\psi \models \psi \theta$.

\section{Downward Refinement Operator}

Let the search space $S$ be the set of all PCNF of a first order logical language which has a finite number of function and predicate symbols. Let the top $\top \in S$ be the conjunction of a positive number of empty clauses, i.e. $T=\square \wedge \square \ldots \wedge$ $\square$. The number of $\square$ is irrelevant because they are all equivalent to false. A refinement operator on $S$ is a function $\rho: S \rightarrow 2^{S}$ (the set of all subsets of $S)$. A refinement operator $\rho$ is downward if for every $\psi$ and $\phi \in \rho(\psi)$, we have $\psi \models \phi$. A refinement chain from $\psi$ to $\phi$ is a sequence $\psi_{0}, \psi_{1}, \ldots, \psi_{k}$ in $S$ such that $\psi_{0} \Leftrightarrow \psi, \psi_{k} \Leftrightarrow \phi$ and $\psi_{i} \in \rho\left(\psi_{i-1}\right)$.

Definition 7. Let $\psi=q_{1} x_{1} \ldots q_{n} x_{n} C_{1} \wedge C_{2} \ldots \wedge C_{m}$. Let $\rho$ be a refinement operator on $S$ defined by the following items.

Note that there are only a finite number of non-alphabetical variants in $\rho(\psi)$.

The first three items have to do with universal variables:

- 1. For an elementary u-unification $\theta=\{y / x\}$, where $x, y \in u \operatorname{Var}(\psi)$ and $x$ comes before $y$ in $Q(\psi)$, let $\psi \theta \in \rho(\psi)$.

- 2. Let $x \in \mathrm{u} \operatorname{Var}(\psi)$ and an elementary u-substitution $\theta=\left\{x / f\left(y_{1}, \ldots, y_{k}\right)\right\}$, let $\psi \theta \in \rho(\psi)$.

- 3. Let $L=p\left(y_{1}, \ldots, y_{k}\right)$ or $\neg p\left(y_{1}, \ldots, y_{k}\right)$ where $y_{1}, \ldots, y_{k}$ are distinct variables new w.r.t. $\psi$. For an arbitrary $j=1, \ldots, m$, let $\phi_{j}$ be defined by adding a u-literal to $\psi: \phi_{j}=q_{1} x_{1} \ldots q_{n} x_{n} \forall y_{1} \ldots \forall y_{k} C_{1} \wedge \ldots \wedge\left(C_{j} \vee L\right) \wedge \ldots \wedge C_{m}$. Then $\phi_{j} \in \rho(\psi)$.

The next 3 items have to do with existential variables:

- 4. Let $x \in \mathrm{eVar}(\psi)$ and $\left\{\left(x, p_{1}\right), \ldots,\left(x, p_{k}\right)\right\}$ be some (not all) $x$-occurrences in $M(\psi)$. For a new variable $y$ and an elementary e-antiunification $\theta=$ $\left\{\left(x / y, p_{1}\right), \ldots,\left(x / y, p_{k}\right)\right\}$, let $\psi \theta \in \rho(\psi)$.

- 5. Let $\left(t, p_{i}\right)=\left(f\left(y_{1}, \ldots, y_{s}\right), p_{i}\right), i=1, \ldots, k$ be term occurrences in $M(\psi)$ such that all $y_{j}$ are distinct and in $\mathrm{eVar}(\psi)$. Then for the elementary esubstitution $\theta=\left\{\left(t / y, p_{1}\right), \ldots,\left(t / y, p_{k}\right)\right\}$, let $\psi \theta \in \rho(\psi)$.

- 6. Let $L=p(y, \ldots, y)$ or $L=\neg p(y, \ldots, y)$ be a literal with new variable $y$. Then for $j=1, \ldots, m$, let $\phi_{j} \in \rho(\psi)$ be defined by adding an e-literal: $\phi_{j}=\exists y q_{1} x_{1} \ldots q_{n} x_{n} C_{1} \wedge \ldots \wedge\left(C_{j} \vee L\right) \ldots \wedge C_{m}$.

The last item has to do with eu-substitutions:

- 7. Let $x \in \mathrm{e} \operatorname{Var}(\psi)$ and $y \in \mathrm{u} \operatorname{Var}(\psi)$ and suppose $x$ comes before $y$ in $Q(\psi)$. For the elementary eu-substitution $\theta=\{(x, y)\}$, let $\psi \theta \in \rho(\psi)$

Theorem 3. If $\phi \in \rho(\psi)$, then $\psi \models \phi$, i.e. $\rho$ is a downward refinement operator. 


\section{The Completeness of the Refinement Operator $\rho$}

In this section we will show that the refinement operator $\rho$ is weakly complete in $S$. That is to say, for every $\phi$ in $S$, there is a finite refinement chain from $T$ to $\phi$. We show this by the following steps. Example 3 will illustrate these steps more concretely.

1. Replace every existential variable in $M(\phi)$ by a new constant not in $\phi$ and remove all the existential variables from $Q(\phi)$. Let the new PCNF be $\psi$. Then the variables in $\psi$ are universally quantified. Let $M(\psi)=C_{1} \wedge C_{2} \ldots \wedge C_{m}$. We have then $\square$ subsumes $C_{i}\left(\square \succeq C_{i}\right)$ for all $i$.

2. Similar to a result about the classic refinement refinement operator [S81,NW97], we can prove that there is a chain from $\square$ to every $C_{i}$. The combination of these chains will give a chain from $T$ to $\psi$.

3. By using the elementary e-substitutions (item 4 of $\rho$ ) we can change the constant occurrences in $\psi$ back to existential variables. This establishes a refinement chain from $\psi$ to $\phi^{\prime}$ which looks almost like $\phi$ but all existential variables appear before the universal variables.

4. Using eu-substitutions (item 7 of $\rho$ ) we can move the existential variables to the right places in the prenex. This means there is a chain from $\phi^{\prime}$ to $\phi$. Thus we have the weak completeness of $\rho$.

Example 3. We will give an example to show what a concrete finite chain from $\top$ to a given $\phi=\forall x \exists y((\neg p(x) \vee q(f(x)) \vee q(y)) \wedge r(y, a))$ looks like. Note that the chain is not unique. Such a chain exists for a general $\phi$ (Theorem 4 ) because of the following lemmas. We use an arrow $\stackrel{n}{\longrightarrow}$ to denote a refinement step which uses the $n$-th item of $\rho$.

$$
\begin{aligned}
& \square \stackrel{3}{\longrightarrow} \forall x \neg p(x) \stackrel{3,3,3}{\longrightarrow} \\
& \forall x \forall u \forall v \forall w \forall w^{\prime}\left((\neg p(x) \vee p(u) \vee q(v)) \wedge r\left(w, w^{\prime}\right) \stackrel{2}{\rightarrow}\right. \\
& \forall x \forall u_{1} \forall v \forall w \forall w^{\prime}\left(\left(\neg p(x) \vee p\left(f\left(u_{1}\right)\right) \vee q(v)\right) \wedge r\left(w, w^{\prime}\right) \stackrel{1,1}{\longrightarrow}\right. \\
& \forall x \forall v \forall w^{\prime}(\neg p(x) \vee p(f(x)) \vee q(v)) \wedge r\left(v, w^{\prime}\right) \stackrel{2,2}{\rightarrow} \\
& \psi=\forall x(\neg p(x) \vee p(f(x)) \vee q(b)) \wedge r(b, a)) \stackrel{5}{\rightarrow} \\
& \phi^{\prime}=\exists y \forall x(\neg(p(x) \vee p(f(x)) \vee q(y)) \wedge r(y, a) \stackrel{7}{\rightarrow} \\
& \phi=\forall x \exists y(\neg p(x) \vee p(f(x)) \vee q(y)) \wedge r(y, a)
\end{aligned}
$$

Lemma 6. Let $C$ be a universally quantified clause. Then there is a finite chain of refinements from $\square$ to $C$.

Lemma 7. Let $\psi=Q(\psi) M(\psi)$ be a universally quantified PCNF. Then there is a finite $\rho$-chain from $\mathrm{T}$ to $\psi$.

Lemma 8. Let $\phi=\exists y_{1} \ldots \exists y_{n} \forall x_{1} \ldots \forall x_{m} M(\phi)$ whose existential variables in the prenex appear before the universal variables. Let $b_{1}, \ldots, b_{n}$ be different constants which do not appear in $\phi$. Let the universally quantified $\psi$ be $\phi$ after replacing variable $y_{i}$ by $b_{i}$. Then there is a finite $\rho$-chain from $\psi$ to $\phi$.

Theorem 4. Given $\phi=Q(\phi) M(\phi)$, there is a finite $\rho$-chain from $\top$ to $\phi$. 


\section{Learning PCNF in Practice}

Based on the refinement operator given in the previous sections, we have extended a simple version of the Claudien system (see [RD97]) to a learning system PCL (abbreviation of PCNF Claudien), and implemented it in Prolog. Learning by interpretations[R97] is used. Claudien learns a universal clausal theory w.r.t. a set of positive examples (interpretations), such that each example is a model of the theory. This clausal theory can be considered as a set of regularities (or integrity constraints) satisfied by these examples. PCL is able to learn a PCNF which is more expressive.

Consider a finite set of scenes (bongard interpretations) as positive examples. A scene contains several figures: each figure has properties like shape, size,... and these figures are related to each other indicated by in, above, left_of.... Claudien is able to find things like: $\forall x \forall y($ shape $(y$, circle $) \leftarrow$ figure $(x)$, figure $(y)$, in $(x, y))$, i.e., for every figure $x$ and $y$ in a scene, when $x$ is inside $y$, then $y$ must be a circle. The following rule cannot be found by Claudien, but PCL can: $\forall x \exists y((\operatorname{in}(x, y) \leftarrow \operatorname{shape}(x$, triangle $)) \wedge$ shape $(y$, circle $))$, each triangle is in at least one circle.

To extend Claudien towards PCL, several issues have to be addressed. In the following subsections we discuss the most important ones.

\subsection{Testing interpretation in a search space}

In Claudien, a clause head $\leftarrow$ body is true for an example if the Prolog query ? - body, not(head) fails for that example. This works only if the clauses are range restricted, meaning that all variables in the head should also occur in the body. Indeed, consider a Herbrand interpretation $I=\{p(a, b), q(a)\}$ and $\phi=\forall x \forall y(p(x, y) \leftarrow q(x)) . \phi$ is false because $p(a, a) \leftarrow q(a)$ is false. We get $N o$ as the answer of the query because there is a refutation of false $\leftarrow p(x, y)$ and $p(x, y) \leftarrow q(x)$.

For PCL, we also consider range restricted PCNFs. The following definition can be found in [N82]: a PCNF $\phi$ in $S$ is range restricted iff

- If $x \in \mathrm{u} \operatorname{Var}(\phi)$ and $x$ is in a positive literal of a clause $C$ (in head $(C)$ ) in $M(\phi)$, then $x$ must also appear in a negative literal of $C$ (in $\operatorname{bod} y(C)$ ).

- If $x \in \mathrm{eVar}(\phi)$ and $x$ is in a negative literal of a clause $C$ (in $\operatorname{bod} y(C)$ ) in $M(\phi)$, then there is a clause $D$ in $M(\phi)$ with only positive literals (no body) such that $x$ appears in every literal in $D$ (in every atom of head $(D)$ ).

Intuitively, a range restricted formula is structured in such a way that for an interpretation, the range of a variable is restricted to the elements defined by some other relations in the same formula. For example, $\exists x($ false $\leftarrow p(x)))$ is not range restricted. To test this kind of rule, we need an explicit domain for the variable $x$. Let $\phi=\forall x \exists y(r(y) \wedge(q(x) \leftarrow p(x, y)))$ and $I=\{q(a), r(a), p(b, a)\}$. Then $\phi$ is range restricted. To check if $I$ is a model of $\phi$, we need only to consider $y$ where $r(y)$ is true, i.e. $\{y / a\}$. 
A search space $S$ for PCL consists of range restricted, function free PCNFs (the only functions are constants). Moreover, in a PCNF there are upper bounds for clauses in a matrix, literals in each clause, constants and variables, hence $S$ is a finite set. The search space is further restricted by some language bias. For instance, the types of the arguments of each predicate must be declared. Also, a declaration is necessary for each type for which constants must be generated. This allows for example to specify some types where no constants should be generated,

\subsection{The downward refinement operator}

For the implementation of the refinement operator $\rho$, several issues must be addressed

First, for efficiency it is necessary to optimize the refinement operator. We should try to avoid deriving equivalent PCNF. One way to do this is to define an order in which literals occur in each clause and an order of the clauses. This removes equivalencies obtained by applying assiociativity and commutativity rules. e.g. we can obtain false $\leftarrow p \wedge q$ in two ways: We can start from the empty clause false, first add $p$ to obtain false $\leftarrow p$ and then add $q$ to obtain false $\leftarrow p \wedge q$. We could also first add $q$ to obtain false $\leftarrow q$ and then add $p$ to obtain false $\leftarrow q \wedge p$ but the latter is not allowed because $p$ comes alphabetically before $q$. Such orders are also considered in Claudien using the DLAB language bias.

Second, we need to address the following problem: For every $i$-th clause in a matrix with $n(\geq i)$ clauses, we can start from the conjunction of $i$ empty clauses Thus we have to search $n$ trees. The computational cost of searching in the $i$-th tree grows very fast as $i$ increases. We can solve this problem partially by reusing the formulas we have found. If $q_{1} x_{1} \ldots q_{k} x_{k} C_{1} \wedge \ldots \wedge C_{i-1} \wedge C_{i}$ is a PCNF with $i$ clauses which is correct w.r.t. the examples, then $q_{1} x_{1} \ldots q_{k} x_{k} C_{1} \wedge \ldots \wedge C_{i-1}$ must also be a correct PCNF. To find the correct PCNF with $i$ clauses, we can start from a correct PCNF containg $i-1$ clauses with an extra empty clause added. The refinement operator can be applied again and again until good PCNFs are found.

\section{$7 \quad$ Experiments}

In this section we present some experiments which illustrate PCL and show that PCL can learn rules which can not be learned by existing systems that only learn single clauses.

Experiment 1 We considered a set of undirected graphs as positive examples. A possible example:

$r(a, b) . \quad r(b, a) \cdot r(a, c) \cdot r(c, a)$.

$r(a, d) . \quad r(d, a) . \quad r(e, a) . \quad r(a, e)$. 
Every graph had the property that each point was connected to at least one other point. PCL found this and also some other rules:

$\forall x \forall y(r(x, y) \leftarrow r(y, x)), \quad \forall x \exists y(r(x, y) \leftarrow \operatorname{point}(x))$

Note that the last PCNF is made range restricted by adding a predicate point. PCL automatically makes PCNF formulas range restricted by using the language bias and the definitions of the domain predicates.

Experiment 2 We also did an experiment on some bongard-like examples mentioned at the beginning of last section. These are scenes of figures (in this case triangles and circles) which are related to each another. One example is

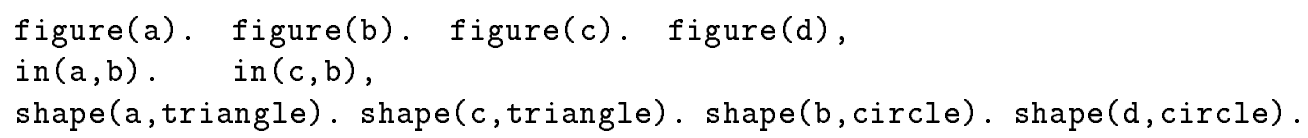

The search space is large and many correct formulas are possible. This means that, even more than the case of Claudien, many trivial rules are found which do not give much new knowledge:

$$
\begin{aligned}
& \exists x(\text { shape }(x, \text { triangle }), \quad \exists x \exists y(\operatorname{in}(x, y)), \\
& \exists y(\text { figure }(y) \wedge(\text { false } \leftarrow \text { shape }(y, \text { triangle }))) .
\end{aligned}
$$

After the search continued for some time, more interesting results were given, such as: $\forall x \exists y$ (shape $(y$, circle $) \wedge(\operatorname{in}(x, y) \leftarrow \operatorname{shape}(x$, triangle $))$.

\section{Conclusion and Future Work}

As we know, every closed formula is equivalent to a PCNF but not necessarily its Skolem standard form. Until now we consider in ILP almost exclusively conjunctions of universally quantified clauses, especially Horn clauses. To add expressiveness we should consider PCNF in general.

If we want to extend refinement operators from set of clauses to set of PCNF, we should first extend substitutions to PCNF. In this article we have defined the substitutions which specialize a given PCNF. Elementary substitutions and adding literals can be used to define a refinement operator $\rho$ which is weakly complete. Notice that we have not used items 5 and 6 in $\rho$ for weak completeness. In a set of formulas ordered by some kind of generalization, a refinement operator is complete if there is a refinement chain from $\psi$ to $\phi$ whenever $\psi$ is more general than $\phi$. For example, item 5 is needed when we consider $\psi=\exists x p(x, x)$ and $\phi=\exists x \exists y p(x, y)$. We would like to know more about the search spaces where $\rho$ is complete and the relation between item 5,6 and the completeness.

This article lays a theoretical foundation for a system we would like to implement which generalizes the Claudien learning system. In fact, a simple system PCL is already implemented which deals with a finite search space of function free and range restricted PCNF. 


\section{References}

[R97] L. De Raedt, Logical settings for concept learning, AI Journal, 95:187-201, 1997.

[RD97] L. De Raedt and L. Dehaspe, Clausal discovery, Machine Learning, 26:99146, 1997.

[GF96] M. Goncalves and C. Froidevaux, A new formalism to integrate quantification in inductive processes, Proceedings of ILP96, S. Muggleton (ed.) Vol. 1314 of LNAI series, 1997, Springer, Berlin.

[LN94] P. van der Laag and S. H. Nienhuys-Cheng, Existence and nonexistence of complete refinement operators, Proceedings of ECML94, Vol. 784 of LNAI series, F. Bergadano and L. De Raedt (eds.). Springer-Verlag, Berlin, 1994.

[N82] J.-M. Nicolas, Logic for Improving Integrity Checking in Relational Data Bases, Informatica, 1982, Springer-Verlag.

[NLR99] S. H. Nienhuys-Cheng, W. Van Laer, L. De Raedt, Substitutions and Refinement operator for PCNF, Work Report, EUR-FEW-CS-99-03.

[NW97] S. H. Nienhuys-Cheng and R. de Wolf, Foundations of Inductive Logic Programming, LNAI Tutorial 1228, Springer-Verlag, 1997.

[S81] E. Y. Shapiro, Inductive inference of theories from facts. Research Report 192, Yale University, 1981. 